\title{
Estado Nutricional de lodo na Gestação e sua Influência na Saúde do Binômio Mãe-Filho
}

\author{
Karen Thayane de Oliveira Coqueiro, ${ }^{1}$ Renata Junqueira Pereira ${ }^{2}$
}

\section{RESUMO}

A gravidez está associada a um declínio do iodo no organismo materno, que pode levar ao hipotireoidismo em áreas com deficiência desse elemento. Por esta razão, é fundamental uma ingestão adequada deste nutriente durante a gravidez. Verifica-se que a insuficiente ingestão materna de iodo afeta negativamente a saúde do feto e do recém-nascido, com consequências que perdurarão por toda a vida. Esta revisão teve como objetivo descrever a importância do iodo para o período gestacional e os efeitos relacionados à sua deficiência no binômio mãe-filho. Sabe-se que o iodo é essencial para o desenvolvimento do cérebro fetal em etapas tais como a neurogênese, a diferenciação de células neuronais e gliais, a mielinização, a migração e a sinaptogênese. Foi realizada uma revisão bibliográfica, com buscas nas principais bases de dados em saúde, como Medline (National Library of Medicine, Estados Unidos), via PubMed, Lilacs (Literatura Latino-Americana e do Caribe em Ciências da Saúde), SciELO (Scientific Eletronic Library Online) e em trabalhos publicados entre 2009 e 2020, nos idiomas português, inglês e espanhol. Futuros estudos sobre o papel da deficiência iódica na saúde materna e fetal ainda são necessários para elucidar de que forma o iodo impacta nos desfechos gestacionais e perinatais e as possibilidades de intervenção para se evitar ou minimizar os efeitos indesejáveis de sua deficiência.

Palavras-chave: Deficiência de iodo. Nutrição da gestante. Complicações na gravidez.

NUTRITIONAL STATUS OF IODINE IN PREGNANCY AND ITS INFLUENCE ON THE MOTHER-CHILD BINOMIAL HEALTH ABSTRACT

Pregnancy is associated with a decline in iodine in the maternal body, which can lead to hypothyroidism in iodine deficient areas. For this reason, an adequate intake of this nutrient during pregnancy is essential. Insufficient maternal iodine intake negatively affects the health of the fetus and newborn, with consequences that will last a lifetime. This review aimed to describe the importance of iodine for the gestational period and the effects related to its deficiency in the mother-child binomial. lodine is known to be essential for fetal brain development in stages such as neurogenesis, neuronal and glial cell differentiation, myelination, migration and synaptogenesis. An integrative literature review was performed, searching the main health databases Medline (National Library of Medicine, United States), via PubMed, Lilacs (Latin American and Caribbean Health Sciences Literature), SciELO (Scientific Electronic Library) Online), of works published between 2009 and 2020, in the languages: Portuguese, English and Spanish. Future studies on the role of iodine deficiency in maternal and fetal health are still needed to clarify how iodine impacts gestational and perinatal outcomes and the possibilities for intervention to prevent or minimize the undesirable effects of iodine deficiency.

Keywords: lodine deficiency. Nutrition of pregnant women. Complications in pregnancy.

\footnotetext{
${ }^{1}$ Universidade Federal do Tocantins, Campus de Palmas. Palmas/TO, Brasil.

${ }^{2}$ Autora correspondente. Universidade Federal do Tocantins - Quadra 109 Norte, Avenida NS15, ALCNO14, Campus de Palmas. Palmas/TO, Brasil. http:// lattes.cnpq.br/5178647720304213. http://orcid.org/0000-0001-9487-4013. renatajunqueira@uft.edu.br
} 


\section{INTRODUÇÃO}

A adequada nutrição materna durante a gestação representa um desafio, uma vez que as necessidades nutricionais tornam-se aumentadas nesse período e qualquer alteração na ingestão de nutrientes materna pode afetar a saúde materno-fetal. As deficiências de micronutrientes em gestantes estão ligadas à pré-eclâmpsia, aborto, retardo de crescimento intrauterino e anormalidades congênitas (GARCÍA et al., 2016).

$O$ iodo é um constituiente primordial dos hormônios da tireoide, a 3,5,3-triiodo-L-tironina (T3) e a 3,5,3,5-tetraiodo-L-tironina (T4), importantes para o crescimento, desenvolvimento e metabolismo saudáveis. Adequados níveis de iodo durante a gestação são essenciais para o neurodesenvolvimento fetal e a leve deficiência de iodo está relacionada a prejuízos nesse desenvolvimento (COMBET et al., 2015).

A literatura evidencia que durante o período gestacional ocorrem profundas alterações imunológicas e hormonais, revelando-se comuns disfunções na tireoide nesse período, que podem trazer complicações ao feto, dependente do iodo e dos hormônios maternos (BUSINGE; LONGO-MBENZA; KENGNE, 2019) Devido aos ajustes fisiológicos da gestação, as necessidades de iodo aumentam de $150 \mu \mathrm{g} / \mathrm{dia}$ em mulheres adultas não grávidas para $250 \mu \mathrm{g} / \mathrm{dia}$ em gestantes (MOLINA et al., 2015). Durante a gestação a ingestão de iodo deve ser aumentada para atender às demandas de produção e transferência placentária de hormônios tireoidianos, primordiais para um desenvolvimento fetal adequado (EASTMAN; MA; LI, 2019).

O feto torna-se capaz de produzir hormônios da tireoide somente durante a segunda metade do período gestacional, por isso depende integralmente da concentração de iodo circulante na mãe durante toda a gravidez e dos níveis de hormônios tireoidianos maternos durante os primeiros meses (MARKHUS et al., 2018).

Os hormônios da tireoide participam de estágios críticos da formação cerebral durante as fases embrionárias, fetal e pós-natal. A deficiência iódica na gestação ocasiona danos cerebrais irreversíveis, manifestados por déficit cognitivo, distúrbios comportamentais e cretinismo neurológico (EASTMAN; MA; LI, 2019). A principal causa evitável de dano cerebral (retardo mental e paralisia cerebral) em todo o mundo é a deficiência de iodo (MOLINA et al., 2012; BOTTARO et al., 2016).

Entre as consequências relacionadas à deficiência do iodo durante o período gestacional também estão a ocorrência de natimortos, abortos, alterações psicomotoras, aumento da mortalidade perinatal, anormalidades congênitas, hipotireoidismo congênito e bócio neonatal (HENRICHS et al., 2010; MIN et al., 2016).

Nesse sentido, o presente estudo teve como objetivo revisar a literatura para descrever a importância do iodo no período gestacional e os efeitos relacionados à sua deficiência no binômio mãe-filho.

\section{METODOLOGIA}

Este trabalho teve como questão norteadora o consumo de iodo durante a gestação e qual a relação da deficiência deste para a saúde do binômio mãe e filho. Foi realizada uma revisão bibliográfica, com buscas nas principais bases de dados em saúde, tais como Medline (National Library of Medicine, Estados Unidos), via PubMed, Lilacs (Literatura Latino-Americana e do Caribe em Ciências da Saúde), SciELO (Scientific Eletronic Library Online) e trabalhos publicados nos últimos anos nos idiomas: português, inglês e espanhol. Foi estabelecido um marco temporal de 2009 a 2020 com estudos para compor a revisão dos últimos 11 anos. Também foi utilizado o critério de busca na literatura cinzenta, em que se pesquisa as citações da lista de referências dos estudos selecionados.

Foram utilizados os descritores gestação, iodo, deficiência de iodo (em português, inglês e espanhol) combinados, para que a busca ficasse mais objetiva, ficando assim: gestação e iodo; gestação e deficiência de iodo; em inglês, segundo o Medical Subject Headings (MeSH) e em português, conforme os Descritores em Ciências da Saúde (DeCS). A busca dos artigos que compuseram este estudo foi realizada entre os meses de junho e outubro do ano 2020. Foram incluídos apenas os artigos com disponibilidade de texto completo e originais, e estudos disponíveis nas bases de dados selecionadas. Foram excluídos artigos que não abordavam a deficiência iódica na gestação e suas consequências, artigos de revisão, editoriais, cartas ao editor e estudos que não analisaram a temática relevante ao objetivo da revisão.

\section{RESULTADOS E DISCUSSÃO}

Foram encontrados um total de 1.256 artigos, dos quais foram selecionados 28 para a abordagem neste estudo. A maior parte apresentou efeitos da deficiência do iodo relacionados ao feto, porém foram encontrados poucos estudos relacionados aos efeitos da deficiência do iodo na saúde materna.

De acordo com Milagres et al. (2020), a deficiência de iodo é encontrada em um terço da população mundial, nos grupos mais vulneráveis, como as 
mulheres grávidas e as crianças. Além disso, as diferenças nos teores de iodo dos alimentos - visto que características climáticas e geográficas interferem no seu conteúdo - somadas aos distintos hábitos alimentares, afetam diretamente o estado nutricional desse nutriente na população. Ainda segundo esses autores, no Brasil não há a quantificação do teor de iodo dos alimentos nacionais, o que dificulta a avaliação do seu consumo pela população.

Sabe-se que o iodo é essencial para o desenvolvimento do cérebro fetal em etapas tais como a neurogênese, a diferenciação de células neuronais e gliais, a mielinização, a migração e a sinaptogênese. Nesse sentido, a deficiência de iodo na gestação e na vida extrauterina pode ocasionar um conjunto de anormalidades denominadas distúrbios da deficiência de iodo (LEVIE et al., 2018).

Os distúrbios da deficiência de iodo incluem um espectro de diferentes doenças, como hipotireoidismo, natimortos, bócio, anomalias congênitas, crescimento e desenvolvimento infantis prejudicados, atraso no desenvolvimento psicomotor, redução da capacidade cognitiva, além de elevarem as taxas de mortalidade fetal e infantil e serem considerados as principais causas evitáveis de deficiência mental na infância e cretinismo (CHOUDHRY; NASRULLAH, 2018).

Estudos animais revelam danos no desenvolvimento neurológico induzidos por hipotiroxinemia materna, com limitação do crescimento dendrítico e axonal, localização neural anormal e alterações da função sináptica (MIN et al., 2016).

Uma metanálise, realizada por Bougma et al. (2013), estudou a deficiência de iodo no desenvolvimento intelectual de crianças e verificou a ocorrência de problemas neurocognitivos, ao constatar o Quociente de Inteligência de filhos de mães insuficientes em iodo, 7,4 pontos mais baixos que os de filhos de mães suficientes. Choudhry e Nasrullah (2018) enfocaram aspectos do consumo adequado de iodo na gestação para evitar comprometimentos cognitivos na criança e concluíram que a inclusão adequada de alimentos fontes de iodo na alimentação regular é essencial para a regulação das funções do corpo humano, em todas as faixas etárias. Segundo esses autores, garantir suficientes ofertas de iodo à população em geral é necessário para que se alcance um perfeito desempenho cognitivo.

Em estudo semelhante, realizado por Hay, Hynes e Burgess (2019), reforça-se que a deficiência de iodo durante o período gestacional está relacionada ao comprometimento do desenvolvimento do sistema nervoso central fetal durante o primeiro trimestre da gravidez. Os autores concluem que as mulheres em idade fértil, que consideram a chance de engravidarem, e que residem em áreas onde há a deficiência de iodo, devem ser orientadas a tomarem suplementação desse elemento, por vários meses, antes, durante e após a gravidez.

Para Levie et al. (2018), tanto o hipotireoidismo quanto os baixos níveis séricos de T4 livre materno estão associados a um menor Quociente de Inteligência, menor volume cerebral, maior risco de transtornos do espectro autista, prejuízo da função psicomotora e esquizofrenia.

Estudo realizado por Markhus et al. (2018) demonstrou que níveis de níveis urinários de iodo maternos inferiores a $100 \mu \mathrm{g} / \mathrm{L}$ estiveram associados à redução das habilidades de linguagem receptiva e expressiva, mas não à diminuição de habilidades cognitivas e motoras em crianças até 18 meses. Esses mesmos autores não evidenciaram efeito protetor dos suplementos contendo iodo quando usados na gravidez.

Estudo recente realizado por Robinson et al. (2018) concluiu que baixos níveis de iodo materno no período periconcepção podem afetar o Quociente de Inteligência na infância.

Pesquisa realizada por Henrichs et al. (2010) associou a hipotiroxinemia materna leve e grave a um maior risco de atraso da linguagem expressiva em todas as idades, e a hipotiroxinemia materna grave a maior risco de atraso cognitivo não verbal. Esses autores também evidenciaram que os níveis de hormônio estimulante da tireoide (TSH) não estiveram associados à cognição das crianças.

Outros estudos também já demonstraram a ausência de correlação entre os níveis de TSH do recém-nascido e o estado nutricional de iodo materno (MIRANDA; SOUSA, 2018; LEVIE et al., 2018).

De acordo com Candido et al. (2020), o momento gestacional mais afetado pela deficiência de iodo são o segundo e o terceiro trimestres, observando-se então maior risco de nódulos pneumáticos, hipotireoidismo subclínico e pré-eclâmpsia. Os mesmos autores concluíram que os danos causados pela deficiência de iodo no primeiro ou segundo trimestres parecem reversíveis, no entanto necessitam de diagnóstico preciso e precoce para prevenção de danos à saúde do binômio mãe-filho.

Miranda e Sousa (2018) postulam que, apesar de o nível de TSH sérico ser o indicador mais preciso da função tireoidiana na gravidez, o nível sérico de T4 livre materno, no primeiro trimestre de gravidez, atua como principal determinante do desenvolvimento psi- 
comotor pós-natal. Mesmo um período transitório de hipotiroxinemia materna, no início da neurogênese fetal, pode aumentar o risco de atrasos na linguagem expressiva e nas funções cognitivas não verbais da criança.

No que se refere ao período perinatal, estudo de Léon et al. (2015) demonstrou associação inversa entre os níveis de T4 livre e TSH maternos e o peso ao nascer da criança, observando-se que elevados níveis de T4 livre foram associados a risco aumentado de nascer pequeno para a idade gestacional (PIG). 0 peso médio ao nascer foi maior no grupo hipotireoxêmico e a ingestão de iodo materna, bem como a UIC não se associaram aos desfechos estudados ao nascer. Os autores ressaltam que os níveis de hormônios tireoidianos maternos podem impactar no desfecho da gestação, mesmo dentro dos valores considerados normais. Resultados semelhantes foram também encontrados por Medici et al. (2013) para a ocorrência de baixo peso ao nascer e nascimentos PIG.

No tocante à saúde materna, Morchiladze et al. (2017) destacaram que gestantes com hipotiroxinemia isolada demonstraram maior prevalência de sinais e sintomas como astenia, pele seca, aumento de queda de cabelo e fragilidade de unhas, bem como de distúrbios associados à gestação, como vômitos e anemia. Outro estudo constatou também que o risco prognóstico de aborto espontâneo precoce, parto prematuro e intervenções cirúrgicas obstétricas foi maior em gestantes com hipotireoidismo. Além disso, foi observada também maior proporção relativa de baixo peso ao nascer, anemia materna por deficiência de ferro no pós-parto, ganho de peso anormal e distúrbios venosos crônicos dos membros inferiores nessas gestantes (MORCHILADZE et al., 2017).

Um estudo brasileiro, avaliando gestantes do estado mais populoso do país (São Paulo), constatou que as gestantes se apresentaram levemente deficientes em iodo, com nível mediano de $\mathrm{UCl}$ em 137,7 $\mu \mathrm{g} / \mathrm{L}$, apesar de utilizarem de forma contínua o sal de cozinha iodado, amplamente comercializado no Brasil, como única fonte de iodo da dieta (FERREIRA et al., 2014). Estudos já demonstraram que uma deficiência de iodo leve a moderada prejudica a cognição (BOUGMA et al., 2013; COMBET et al., 2015; CHOUDHRY; NASRULLAH, 2018).

Estudos realizados por Ferreira et al. (2014) e Bottaro et al. (2016) analisaram o estado nutricional de iodo em gestantes, por meio da dosagem da concentração urinária de iodo (UIC). Organismos internacionais recomendam a média da UIC como o indicador mais adequado para se avaliar o estado nutricional de iodo na população, posto que mais de $90 \%$ do iodo absorvido é excretado na urina (EASTMAN; MA; LI, 2019).

Os critérios para a avaliação da adequação da ingestão de iodo, tendo por base os valores de iodúria são: UIC <150 é consumo de iodo insuficiente, entre 150-249 adequado, entre 250-499 acima do recomendado e $>500$ é consumo excessivo (EASTMAN; MA; LI, 2019).

Mesmo, no entanto, sem dados robustos acerca do estado nutricional de iodo em gestantes brasileiras, o governo decidiu reduzir a concentração de iodo no sal da alimentação para 15 a $45 \mathrm{mg} / \mathrm{kg}$, com base em observações de que a ingestão de sal dos brasileiros estava elevado, em parte devido ao alto consumo de produtos industrializados (FERREIRA et al., 2014). A concentração ideal de iodo no sal para consumo humano anterior oscilava entre 20 e 60 mg/kg (BRASIL, 2014).

Ademais, a literatura tem mostrado que a iodação do sal tem sido o método mais eficaz de reduzir as prevalências de deficiência iódica, sobretudo quando comparada a estratégias de fortificação de apenas um grupo de alimentos em especial (CLIFTON et al., 2013; BOTTARO et al., 2016).

Fereja et al. (2018) também concluíram sobre os benefícios da iodação do sal para o estado nutricional de iodo em gestantes e reforçam que é necessário fortalecer os programas de iodação de sal como forma de garantir o acesso da população a fontes de iodo, de forma adequada e homogênea. Esses autores também evidenciaram risco até quatro vezes mais elevado de desenvolvimento do bócio em multíparas quando comparadas a nulíparas.

Merece destaque a introdução de produtos alimentícios da moda nas dietas ocidentais, que leva à substituição do sal iodado por outros tipos de temperos, sem iodização adequada, e que chegam ao comércio sem a devida fiscalização pelos órgãos de controle e vigilância e podem afetar o consumo de iodo pela população, uma vez que substituem justamente uma das principais fontes de iodo da alimentação (RAHMAN; ISLAM; FARRUKH, 2015).

Por outro lado, em relação à suplementação de iodo na gestação, Markhus et al. (2018) verificaram que o uso de suplementos contendo iodo não foi associado a efeitos benéficos para o binômio. O excesso de iodo na gestação pode gerar riscos e um estudo com gestantes no Brasil verificou que a excessiva ingestão de iodo durante a gravidez aumentava o risco 
de desenvolver hipertireoidismo subclínico, especialmente em grávidas com concentrações urinárias de iodo superiores a $500 \mu \mathrm{g} / \mathrm{L}$ (CORCINO et al., 2019).

Nessa perspectiva, estudo brasileiro, realizado por Souza et al. (2015), associou a deficiência iódica à hipertensão na gestação, demonstrando que gestantes hipertensas, com concentrações de iodo urinária inferiores a $150 \mu \mathrm{g} / \mathrm{L}$, apresentaram 26\% mais chances de evoluir com deficiência de iodo em comparação às pacientes não hipertensas.

A recomendação para gestantes hipertensas, no entanto, que não estejam hospitalizadas pela sua condição, é dieta normossódica ( $2 \mathrm{~g}$ de sódio/dia) e não implica grande restrição do consumo de sal (OLIVEIRA; FERREIRA; GRACILIANO, 2016). Tal evidência aponta na direção de que a hipertensão possa ser consequência da deficiência iódica e não o contrário.

\section{CONCLUSÃO}

O consumo adequado de iodo durante a gestação é primordial para garantir o adequado desenvolvimento cerebral do feto. A deficiência de iodo durante o período gestacional pode ocasionar danos cerebrais em crianças, pois os hormônios tireoidianos, que são produzidos a partir do iodo, desempenham um papel de fundamental importância no desenvolvimento do sistema nervoso central. Além disso, a deficiência do iodo pode gerar outras condições, como aumento da glândula tireoide (bócio), hipotireoidismo e ganho de peso.

Apesar dos esforços para controlar e prevenir a deficiência iódica durante a gestação, ainda há países em que essa deficiência se destaca como problema de saúde pública, sobretudo durante o período gestacional.

A iodação universal de sal é a principal forma mundialmente aceita e utilizada para aumentar o consumo de iodo, porém como essa recomendação apresenta-se paralela às indicações para diminuição do consumo de sal, é fundamental coordenar as intervenções.

O diagnóstico precoce da deficiência de iodo durante o período gestacional fornece orientação para ingestão adequada, quer dietética ou suplementar, garantindo a homeostase do mineral e a prevenção de danos à saúde materno-infantil.

Estudos controlados e de intervenção, com resultados conclusivos sobre o papel da deficiência iódica na saúde materna e fetal, ainda são escassos. Futuras pesquisas são ainda necessárias para elucidar de que forma o iodo impacta nos desfechos gestacionais e perinatais e as possibilidades de intervenção para se evitar ou minimizar os efeitos indesejáveis de sua deficiência.

\section{REFERÊNCIAS}

BOTTARO, S. et al. Evaluación del estado nutricional de yodo en una población de embarazadas. Rev. Méd. Urug., v. 32, n. 3, p. 152-158, 2016.

BOUGMA, K. et al. lodine and mental development of children 5 years old and under: a systematic review and meta-analysis. Nutrients, v. 5, n. 4, p. 1.384-1.416, 2013.

BRASIL. Agência Nacional de Vigilância Sanitária. Resultado do monitoramento do teor de iodo no sal para consumo humano. Brasília: Anvisa, 2014. 13 p.

BUSINGE, B. C.; LONGO-MBENZA, B.; KENGNE, P. A. The prevalence of insufficient iodine intake in pregnancy in Africa: protocol for a systematic review and meta-analysis. BMC, v. 8, n. 209, p. 2-7, 2019.

CANDIDO, A. C. et al. Implications of iodine deficiency by gestational trimester: a systematic review. Arch Endocrinol. Metab., In press, 2020.

CHOUDHRY, H; NASRULLAH, M. D. lodine consumption and cognitive performance: Confirmation of adequate consumption. Food Sci. Nutr, v. 6, n. 6, p. 1.341-1.351, 2018.

CLIFTON, V. L. et al. The impact of iodine supplementation and bread fortification on urinary iodine concentrations in a mildly iodine deficient population of pregnant women in South Australia. Nutr. J., v. 12, n. 32, 2013.

COMBET, E. et al. lodine and pregnancy - a UK cross-sectional survey of dietary intake, knowledge and awareness. British Journal of Nutrition, v. 114, n. 1, p. 108-117, 2015.

CORCINO, C. M. et al. Variation of iodine status during pregnancy and its associations with thyroid function in women from Rio de Janeiro, Brazil. Public Health Nutr, v. 22, n. 7, p. 1.232-1.240, 2019.

EASTMAN, J. C.; MA, G.; LI, M. Optimal Assessment and Quantification of lodine Nutrition in Pregnancy and Lactation: Laboratory and Clinical Methods, Controversies and Future Directions. Nutrients, v. 11, n. 10, 2019.

FEREJA, M. et al. Prevalence of iodine deficiency and associated factors among pregnant women in Ada district, Oromia region, Ethiopia: a cross- sectional study. BMC Pregnancy and Childbirth, v. 18, n. 257, 2018.

FERREIRA, S. M. S. et al. lodine insufficiency in pregnant women from the State of São Paulo. Arq Bras. Endocrinol. Metab., São Paulo, v. 58, n. 3, p. 282-287, 2014.

GARCÍA, M. M. R. et al. Suplementos en gestación: últimas recomendaciones. Nutr. Hosp., v. 33, n. 4, p. 3-7, 2016.

HAY, I.; HYNES, L. K.; BURGESS, R. J. Mild-to-Moderate Gestational lodine Deficiency Processing Disorder. Nutrients, v. 11, n. 9, 2019.

HENRICHS, J. et al. Maternal thyroid function during early pregnancy and cognitive functioning in early childhood: the generation R study. Journal of Clinical Endocrinology \& Metabolism, v. 95, n. 9, p. 4.227-4.234, 2010. 
LÉON, G. et al. Maternal Thyroid Dysfunction during Gestation, Preterm Delivery, and Birthweight. The Infancia y Medio Ambiente Cohort, Spain. Paediatric and Perinatal Epidemiology, v. 29, n. 2, p. 113-122, 2015.

LEVIE, D. et al. Thyroid Function in Early Pregnancy, Child IQ, and Autistic Traits: A Meta-Analysis of Individual Participant Data, J. Clin. Endocrinol. Metab., v. 103, n. 8, p. 2.9672.979, 2018.

MARKHUS, W. M. et al. Maternal lodine Status is Associated with Offspring Language Skills in Infancy and Toddlerhood. Nutrients, v. 10, n. 9, 2018.

MEDICl, M. et al. Maternal Thyroid Hormone Parameters during Early Pregnancy and Birth Weight: The Generation R Study. J Clin. Endocrinol. Metab., v. 98, n. 1, p. 59-66, 2013.

MILAGRES, R. C. R. M. et al. Food lodine Content Table compiled from international databases. Rev. Nutr., v. 33, e190222, 2020.

MIN, H. et al. Maternal Hypothyroxinemia-Induced Neurodevelopmental Impairments in the Progeny. Mol. Neurobiol., v. 53, n. 3, p. 1.613-1.624, 2016.

MIRANDA, A.; SOUSA, N. Maternal hormonal milieu influence on fetal brain development. Brain and Behavior, v. 8, n. 2, 2018.

MOLINA, C. N. et al. Importance of iodine in pregnancy. Archivos Latino Americanos de Nutrición, v. 62, n. 3, p. 213219, 2012.

MORCHILADZE, N. et al. Prognostic risk of obstetric and perinatal complications in pregnant women with thyroid dysfunction. Georgian Med News, v. 264, p. 21-25, 2017.

MORCHILADZE, N. et al. Importance of isolated gestational hypothyroxinemia in the development of obstetric and somatic pathologies. Georgian Med News, v. 278, p. 39-45, 2018.

OLIVEIRA, A. C. M.; FERREIRA, R. C.; GRACILIANO, N. G. Assistência Nutricional para Gestantes de Alto Risco. In: HORDONHO, A. A. C.; COPPINI, L. Z.; FIDELIX, M. S. P. (org.). Pronutri - Programa de Atualização em Nutrição Clínica: Ciclo 4. Porto Alegre: Associação Brasileira de Nutrição; Artmed Panamericana, 2016. p. 45-102.

RAHMAN, A. U.; ISLAM, A.; FARRUKH, M. A. An Improved Method for the Preparation of Analytical Grade Sodium Chloride from Khewra Rock Salt. World Applied Sciences Journal, v. 8, p. 61-65, 2015.

ROBINSON, S. M. et al. Preconception maternal iodine status is positively associated with iq but not with measures of executive function in childhood. J. Nutr, v. 148, n. 6, p. 959-966, 2018.

SOUZA, L. S. L. et al. Análise do estado nutricional do iodo em gestantes hipertensas e não hipertensas em Salvador Bahia. Revista de Ciências Médicas e Biológicas, Salvador, v. 14, n. 3, p. 323-330, 2015. 